\title{
Doubled patterns are 3-avoidable
}

\author{
Pascal Ochem \\ LIRMM, Université de Montpellier, CNRS \\ Montpellier, France \\ ochem@lirmm.fr
}

Submitted: Oct 7, 2015; Accepted: Jan 18, 2016; Published: Feb 5, 2016

Mathematics Subject Classifications: 68R15

\begin{abstract}
In combinatorics on words, a word $w$ over an alphabet $\Sigma$ is said to avoid a pattern $p$ over an alphabet $\Delta$ if there is no factor $f$ of $w$ such that $f=h(p)$ where $h: \Delta^{*} \rightarrow \Sigma^{*}$ is a non-erasing morphism. A pattern $p$ is said to be $k$-avoidable if there exists an infinite word over a $k$-letter alphabet that avoids $p$. A pattern is said to be doubled if no variable occurs only once. Doubled patterns with at most 3 variables and doubled patterns with at least 6 variables are 3 -avoidable. We show that doubled patterns with 4 and 5 variables are also 3 -avoidable.
\end{abstract}

Keywords: Word; Pattern avoidance.

\section{Introduction}

A pattern $p$ is a non-empty word over an alphabet $\Delta=\{A, B, C, \ldots\}$ of capital letters called variables. An occurrence of $p$ in a word $w$ is a non-erasing morphism $h: \Delta^{*} \rightarrow \Sigma^{*}$ such that $h(p)$ is a factor of $w$. The avoidability index $\lambda(p)$ of a pattern $p$ is the size of the smallest alphabet $\Sigma$ such that there exists an infinite word $w$ over $\Sigma$ containing no occurrence of $p$. Bean, Ehrenfeucht, and McNulty [2] and Zimin [14] characterized unavoidable patterns, i.e., such that $\lambda(p)=\infty$. We say that a pattern $p$ is $t$-avoidable if $\lambda(p) \leqslant t$. For more informations on pattern avoidability, we refer to Chapter 3 of Lothaire's book [8].

It follows from their characterization that every unavoidable pattern contains a variable that occurs once. Equivalently, every doubled pattern is avoidable. Our result is that:

Theorem 1. Every doubled pattern is 3-avoidable.

Let $v(p)$ be the number of distinct variables of the pattern $p$. For $v(p) \leqslant 3$, Cassaigne [5] began and I [10] finished the determination of the avoidability index of every pattern with at most 3 variables. It implies in particular that every avoidable pattern 
with at most 3 variables is 3-avoidable. Moreover, Bell and Goh [3] obtained that every doubled pattern $p$ such that $v(p) \geqslant 6$ is 3-avoidable.

Therefore, as noticed in the conclusion of [11], there remains to prove Theorem 1 for every pattern $p$ such that $4 \leqslant v(p) \leqslant 5$. In this paper, we use both constructions of infinite words and a non-constructive method to settle the cases $4 \leqslant v(p) \leqslant 5$.

Recently, Blanchet-Sadri and Woodhouse [4] and Ochem and Pinlou [11] independently obtained the following.

Theorem 2 ([4, 11]). Let $p$ be a pattern.

(a) If $p$ has length at least $3 \times 2^{v(p)-1}$ then $\lambda(p) \leqslant 2$.

(b) If $p$ has length at least $2^{v(p)}$ then $\lambda(p) \leqslant 3$.

As noticed in these papers, if $p$ has length at least $2^{v(p)}$ then $p$ contains a doubled pattern as a factor. Thus, Theorem 1 implies Theorem 2.(b).

\section{Extending the power series method}

In this section, we borrow an idea from the entropy compression method to extend the power series method as used by Bell and Goh [3], Rampersad [13], and Blanchet-Sadri and Woodhouse [4].

Let us describe the method. Let $L \subset \Sigma_{m}^{*}$ be a factorial language defined by a set $F$ of forbidden factors of length at least 2. We denote the factor complexity of $L$ by $n_{i}=\left|L \cap \Sigma_{m}^{i}\right|$. We define $L^{\prime}$ as the set of words $w$ such that $w$ is not in $L$ and the prefix of length $|w|-1$ of $w$ is in $L$. For every forbidden factor $f \in F$, we choose a number $1 \leqslant s_{f} \leqslant|f|$. Then, for every $i \geqslant 1$, we define an integer $a_{i}$ such that

$$
a_{i} \geqslant \max _{u \in L}\left|\left\{v \in \Sigma_{m}^{i} \mid u v \in L^{\prime}, u v=b f, f \in F, s_{f}=i\right\}\right| .
$$

We consider the formal power series $P(x)=1-m x+\sum_{i \geqslant 1} a_{i} x^{i}$. If $P(x)$ has a positive real root $x_{0}$, then $n_{i} \geqslant x_{0}^{-i}$ for every $i \geqslant 0$.

Let us rewrite that $P\left(x_{0}\right)=1-m x_{0}+\sum_{i \geqslant 1} a_{i} x_{0}^{i}=0$ as

$$
m-\sum_{i \geqslant 1} a_{i} x_{0}^{i-1}=x_{0}^{-1}
$$

Since $n_{0}=1$, we will prove by induction that $\frac{n_{i}}{n_{i-1}} \geqslant x_{0}^{-1}$ in order to obtain that $n_{i} \geqslant x_{0}^{-i}$ for every $i \geqslant 0$. By using (1), we obtain the base case: $\frac{n_{1}}{n_{0}}=n_{1}=m \geqslant x_{0}^{-1}$. Now, for every length $i \geqslant 1$, there are:

- $m^{i}$ words in $\Sigma_{m}^{i}$,

- $n_{i}$ words in $L$,

- at most $\sum_{1 \leqslant j \leqslant i} n_{i-j} a_{j}$ words in $L^{\prime}$, 
- $m\left(m^{i-1}-n_{i-1}\right)$ words in $\Sigma_{m}^{i} \backslash\left\{L \cup L^{\prime}\right\}$.

This gives $n_{i}+\sum_{1 \leqslant j \leqslant i} n_{j} a_{i-j}+m\left(m^{i-1}-n_{i-1}\right) \geqslant m^{i}$, that is, $n_{i} \geqslant m n_{i-1}-\sum_{1 \leqslant j \leqslant i} n_{i-j} a_{j}$.

$$
\begin{aligned}
\frac{n_{i}}{n_{i-1}} & \geqslant m-\sum_{1 \leqslant j \leqslant i} a_{j} \frac{n_{i-j}}{n_{i-1}} & \\
& \geqslant m-\sum_{1 \leqslant j \leqslant i} a_{j} x_{0}^{j-1} & \text { by induction } \\
& \geqslant m-\sum_{j \geqslant 1} a_{j} x_{0}^{j-1} & \\
& =x_{0}^{-1} & \text { by }(1)
\end{aligned}
$$

The power series method used in previous papers $[3,4,13]$ corresponds to the special case such that $s_{f}=|f|$ for every forbidden factor. Our condition is that $P(x)=0$ for some $x>0$ whereas the condition in these papers is that every coefficient of the series expansion of $\frac{1}{P(x)}$ is positive. The two conditions are actually equivalent (Miller [9] uses a similar criterion). The result in [12] concerns series of the form $S(x)=1+a_{1} x+a_{2} x^{2}+a_{3} x^{3}+\ldots$ with real coefficients such that $a_{1}<0$ and $a_{i} \geqslant 0$ for every $i \geqslant 2$. It states that every coefficient of the series $1 / S(x)=b_{0}+b_{1} x+b_{2} x^{2}+b_{3} x^{3}+\ldots$ is positive if and only if $S(x)$ has a positive real root $x_{0}$. Moreover, we have $b_{i} \geqslant x_{0}^{-i}$ for every $i \geqslant 0$.

The entropy compression method as developed by Gonçalves, Montassier, and Pinlou [6] uses a condition equivalent to $P(x)=0$. The benefit of the present method is that we get an exponential lower bound on the factor complexity. It is not clear whether it is possible to get such a lower bound when using entropy compression for graph coloring, since words have a simpler structure than graphs.

\section{Applying the method}

In this section, we show that some doubled patterns on 4 and 5 variables are 3 -avoidable. Given a pattern $p$, every occurrence $f$ of $p$ is a forbidden factor. With an abuse of notation, we denote by $|A|$ the length of the image of the variable $A$ of $p$ in the occurrence $f$. This notation is used to define the length $s_{f}$.

Let us first consider doubled patterns with 4 variables. We begin with patterns of length 9 , so that one variable, say $A$, appears 3 times. We set $s_{f}=|f|$. Using the obvious upper bound on the number of pattern occurrences, we obtain

$$
\begin{aligned}
P(x) & =1-3 x+\sum_{a, b, c, d \geqslant 1} 3^{a+b+c+d} x^{3 a+2 b+2 c+2 d} \\
& =1-3 x+\sum_{a, b, c, d \geqslant 1}\left(3 x^{3}\right)^{a}\left(3 x^{2}\right)^{b}\left(3 x^{2}\right)^{c}\left(3 x^{2}\right)^{d} \\
& =1-3 x+\left(\sum_{a \geqslant 1}\left(3 x^{3}\right)^{a}\right)\left(\sum_{b \geqslant 1}\left(3 x^{2}\right)^{b}\right)\left(\sum_{c \geqslant 1}\left(3 x^{2}\right)^{c}\right)\left(\sum_{d \geqslant 1}\left(3 x^{2}\right)^{d}\right) \\
& =1-3 x+\left(\frac{1}{1-3 x^{3}}-1\right)\left(\frac{1}{1-3 x^{2}}-1\right)\left(\frac{1}{1-3 x^{2}}-1\right)\left(\frac{1}{1-3 x^{2}}-1\right) \\
& =1-3 x+\left(\frac{1}{1-3 x^{3}}-1\right)\left(\frac{1}{1-3 x^{2}}-1\right)^{3} \\
& =\frac{1-3 x-9 x^{2}+24 x^{3}+36 x^{4}-54 x^{5}-108 x^{6}+243 x^{8}+162 x^{9}-243 x^{10}}{\left(1-3 x^{3}\right)\left(1-3 x^{2}\right)^{3}} .
\end{aligned}
$$

Then $P(x)$ admits $x_{0}=0.3400 \ldots$ as its smallest positive real root. So, every doubled pattern $p$ with 4 variables and length 9 is 3 -avoidable and there exist at least $x_{0}^{-n}>2.941^{n}$ ternary words avoiding $p$. Notice that for patterns with 4 variables and length at least 
10, every term of $\sum_{a, b, c, d \geqslant 1} 3^{a+b+c+d} x^{3 a+2 b+2 c+2 d}$ in $P(x)$ gets multiplied by some positive power of $x$. Since $0<x<1$, every term is now smaller than in the previous case. So $P(x)$ admits a smallest positive real root that is smaller than $0.3400 \ldots$ Thus, these patterns are also 3-avoidable.

Now, we consider patterns with length 8 , so that every variable appears exactly twice. If such a pattern has $A B C D$ as a prefix, then we set $s_{f}=\frac{|f|}{2}=|A|+|B|+|C|+|D|$. So we obtain $P(x)=1-3 x+\sum_{a, b, c, d \geqslant 1} x^{a+b+c+d}=1-3 x+\left(\frac{1}{1-x}-1\right)^{4}$. Then $P(x)$ admits $0.3819 \ldots$ as its smallest positive real root, so that this pattern is 3 -avoidable.

Among the remaining patterns, we rule out patterns containing an occurrence of a doubled pattern with at most 3 variables. Also, if one pattern is the reverse of another, then they have the same avoidability index and we consider only one of the two. Thus, there remain the following patterns: $A B A C B D C D, A B A C D B D C, A B A C D C B D$, $A B C A D B D C, A B C A D C B D, A B C A D C D B$, and $A B C B D A D C$.

Now we consider doubled patterns with 5 variables. Similarly, we rule out every pattern of length at least 11 with the method by setting $s_{f}=|f|$. Then we check that

$P(x)=1-3 x+\sum_{a, b, c, d, e \geqslant 1} 3^{a+b+c+d+e} x^{3 a+2 b+2 c+2 d+2 e}=1-3 x+\left(\frac{1}{1-3 x^{3}}-1\right)\left(\frac{1}{1-3 x^{2}}-1\right)^{4}$ has a positive real root.

We also rule out every pattern of length 10 having $A B C$ as a prefix. We set $s_{f}=$ $|f|-|A B C|=|A|+|B|+|C|+2|D|+2|E|$. Then we check that $P(x)=1-3 x+$ $\sum_{a, b, c, d, e \geqslant 1} 3^{d+e} x^{a+b+c+2 d+2 e}=1-3 x+\left(\frac{1}{1-x}-1\right)^{3}\left(\frac{1}{1-3 x^{2}}-1\right)^{2}$ has a positive real root.

Again, we rule out patterns containing an occurrence of a doubled pattern with at most 4 variables and patterns whose reversed pattern is already considered. Thus, there remain the following patterns: $A B A C B D C E D E, A B A C D B C E D E$, and $A B A C D B D E C E$.

\section{Sporadic doubled patterns}

In this section, we consider the 10 doubled patterns on 4 and 5 variables whose 3avoidability has not been obtained in the previous section.

We define the avoidability exponent $A E(p)$ of a pattern $p$ as the largest real $\alpha$ such that every $\alpha$-free word avoids $p$. This notion is not pertinent e.g. for the pattern $A B W B A X A C Y C A Z B C$ studied by Baker, McNulty, and Taylor [1], since for every $\epsilon>0$, there exists a $(1+\epsilon)$-free word containing an occurrence of that pattern. However, $A E(p)>1$ for every doubled pattern. To see that, consider a factor $A \ldots A$ of $p$. If an $\alpha$-free word contains an occurrence of $p$, then the image of this factor is a repetition such that the image of $A$ cannot be too large compared to the images of the variables occurring between the $A$ s in $p$. We have similar constraints for every variable and this set of constraints becomes unsatisfiable as $\alpha$ decreases towards 1 . We present one way of obtaining a lower bound on the avoidability exponent for a doubled pattern $p$ of length $2 v(p)$. We construct the $v(p) \times v(p)$ matrix $M$ such that $M_{i, j}$ is the number of occurrences of the variable $X_{j}$ between the two occurrences of the variable $X_{i}$. Let us show that $A E(p) \geqslant 1+\frac{1}{\beta+1}$ where $\beta$ is the largest eigenvalue of $M$. We consider an occurrence of $p$ and we note $\ell_{i}=\left|A_{i}\right|$. In an $\alpha$-free word, the image of the factor $X_{i} \ldots X_{i}$ of $p$ 
implies that $\frac{2 \ell_{i}+\sum_{1 \leqslant j \leqslant v(p)} M_{i, j} \ell_{j}}{\ell_{i}+\sum_{1 \leqslant j \leqslant v(p)} M_{i, j} \ell_{j}}<\alpha$, that is, $l_{i}<\frac{\alpha-1}{2-\alpha} \sum_{1 \leqslant j \leqslant v(p)} M_{i, j} \ell_{j}$. Thus, the vector $V=\left[\begin{array}{c}\ell_{1} \\ \vdots \\ \ell_{v(p)}\end{array}\right]$ must satisfy $V<\frac{\alpha-1}{2-\alpha} M V$. This implies that the largest eigenvalue $\beta$ of $M$ satisfies $\beta>\frac{2-\alpha}{\alpha-1}$, that is, $\alpha>1+\frac{1}{\beta+1}$. Hence, if $\alpha \leqslant 1+\frac{1}{\beta+1}$, then every $\alpha$-free word avoids $p$. So $A E(p) \geqslant 1+\frac{1}{\beta+1}$.

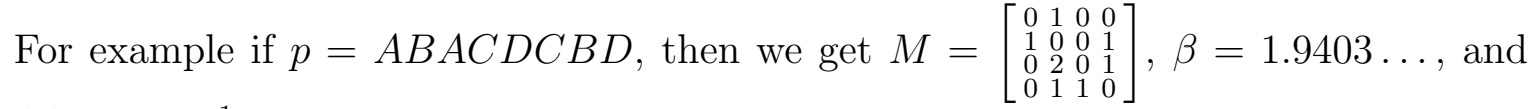
$A E(p) \geqslant 1+\frac{1}{\beta+1}=1.3400 \ldots$. The avoidability exponents of the 10 patterns considered in this section range from $A E(A B C A D B D C) \geqslant 1.292893219$ to $A E(A B A C B D C D) \geqslant$ 1.381966011. For each pattern $p$ among the 10, we give a uniform morphism $m: \Sigma_{5}^{*} \rightarrow \Sigma_{2}^{*}$ such that for every $\left(\frac{5}{4}^{+}\right)$-free word $w \in \Sigma_{5}^{*}$, we have that $m(w)$ avoids $p$. The proof that $p$ is avoided follows the method in [10]. Since there exist exponentially many $\left(\frac{5}{4}^{+}\right)$-free words over $\Sigma_{5}[7]$, there exist exponentially many binary words avoiding $p$.

- $A E(A B A C B D C D) \geqslant 1.381966011,17$-uniform morphism

$$
\begin{aligned}
0 & \mapsto 00000111101010110 \\
1 & \mapsto 00000110100100110 \\
2 & \mapsto 00000011100110111 \\
3 & \mapsto 00000011010101111 \\
4 & \mapsto 00000011001001011
\end{aligned}
$$

- $A E(A B A C D B D C) \geqslant \frac{4}{3}=1.333333333,33$-uniform morphism

$$
\begin{aligned}
& 0 \mapsto 000000101101000111111011001010111 \\
& 1 \mapsto 000000100110100001111101001010111 \\
& 2 \mapsto 000000010110100001111111010010111 \\
& 3 \mapsto 000000010011010100011111010010111 \\
& 4 \mapsto 000000010011001000001111010010111
\end{aligned}
$$

- $A E(A B A C D C B D) \geqslant 1.340090632,28$-uniform morphism

$$
\begin{aligned}
& 0 \mapsto 0000101010001110010000111111 \\
& 1 \mapsto 000000111101000110100111111 \\
& 2 \mapsto 0000001101000011110100100111 \\
& 3 \mapsto 0000001011110000110100111111 \\
& 4 \mapsto 000000101011110010000111111
\end{aligned}
$$

- $A E(A B C A D B D C) \geqslant 1.292893219,21$-uniform morphism

$$
\begin{aligned}
0 & \mapsto 000011101101011111010 \\
1 & \mapsto 000010110100100111101 \\
2 & \mapsto 000001101110100101111 \\
3 & \mapsto 000001101011001111111 \\
4 & \mapsto 000000110111010111111
\end{aligned}
$$


- $A E(A B C A D C B D) \geqslant 1.295597743,22$-uniform morphism

$$
\begin{aligned}
0 & \mapsto 0000011011010100011111 \\
1 & \mapsto 0000011010101001001111 \\
2 & \mapsto 0000001101100100111111 \\
3 & \mapsto 0000001010110000111111 \\
4 & \mapsto 0000000110101001110111
\end{aligned}
$$

- $A E(A B C A D C D B) \geqslant 1.327621756,26$-uniform morphism

$$
\begin{aligned}
0 & \mapsto 00000011110010101011000111 \\
1 & \mapsto 00000011010111111001011011 \\
2 & \mapsto 00000010011111101001110111 \\
3 & \mapsto 00000001001111110001010111 \\
4 & \mapsto 00000001000111111001010111
\end{aligned}
$$

- $A E(A B C B D A D C) \geqslant 1.302775638,33$-uniform morphism

$$
\begin{aligned}
& 0 \mapsto 000000101111110011000110011111101 \\
& 1 \mapsto 000000101111001000001100111111101 \\
& 2 \mapsto 000000011011111001100000100111101 \\
& 3 \mapsto 000000011010101011000001001111101 \\
& 4 \mapsto 000000010111110010101010011111011
\end{aligned}
$$

- $A E(A B A C B D C E D E) \geqslant 1.366025404,15$-uniform morphism

$$
\begin{aligned}
0 & \mapsto 001011011110000 \\
1 & \mapsto 001010100111111 \\
2 & \mapsto 000110010011000 \\
3 & \mapsto 000011111111100 \\
4 & \mapsto 000011010101110
\end{aligned}
$$

- $A E(A B A C D B C E D E) \geqslant 1.302775638,18$-uniform morphism

$$
\begin{aligned}
& 0 \mapsto 000010110100100111 \\
& 1 \mapsto 00001010011111111 \\
& 2 \mapsto 000000110110011111 \\
& 3 \mapsto 000000101010101111 \\
& 4 \mapsto 000000000111100111
\end{aligned}
$$

- $A E(A B A C D B D E C E) \geqslant 1.320416579,22$-uniform morphism

$$
\begin{aligned}
& 0 \mapsto 0000001111110001011011 \\
& 1 \mapsto 0000001111100100110101 \\
& 2 \mapsto 0000001111100001101101 \\
& 3 \mapsto 0000001111001001011100 \\
& 4 \mapsto 0000001111000010101100
\end{aligned}
$$




\section{Simultaneous avoidance of doubled patterns}

Bell and Goh [3] have also considered the avoidance of multiple patterns simultaneously and ask (question 3) whether there exist an infinite word over a finite alphabet that avoids every doubled pattern. We give a negative answer.

A word $w$ is $n$-splitted if $|w| \equiv 0(\bmod n)$ and every factor $w_{i}$ such that $w=$ $w_{1} w_{2} \ldots w_{n}$ and $\left|w_{i}\right|=\frac{|w|}{n}$ for $1 \leqslant i \leqslant n$ contains every letter in $w$. An $n$-splitted pattern is defined similarly. Let us prove by induction on $k$ that every word $w \in \sum_{k}^{n^{k}}$ contains an $n$-splitted factor. The assertion is true for $k=1$. Now, if the word $w \in \sum_{k}^{n^{k}}$ is not itself $n$-splitted, then by definition it must contain a factor $w_{i}$ that does not contain every letter of $w$. So we have $w_{i} \in \Sigma_{k-1}^{n^{k-1}}$. By induction, $w_{i}$ contains an $n$-splitted factor, and so does $w$.

This implies that for every fixed $n$, every infinite word over a finite alphabet contains $n$-splitted factors. Moreover, an $n$-splitted word is an occurrence of an $n$-splitted pattern such that every variable has a distinct image of length 1 . So, for every fixed $n$, the set of all $n$-splitted patterns is not avoidable by an infinite word over a finite alphabet.

Notice that if $n \geqslant 2$, then an $n$-splitted word (resp. pattern) contains a 2 -splitted word (resp. pattern) and a 2-splitted word (resp. pattern) is doubled.

\section{Conclusion}

Our results answer to the first of two questions of our previous paper [11]. The second question is whether there exists a finite $k$ such that every doubled pattern with at least $k$ variables is 2-avoidable. As already noticed [11], such a $k$ is at least 5 since, e.g., $A B C C B A D D$ is not 2-avoidable.

\section{Acknowledgments}

I am grateful to Narad Rampersad for comments on a draft of the paper, to Vladimir Dotsenko for pointing out the result in [12], and to Andrei Romashchenko for translating this paper.

\section{References}

[1] K.A. Baker, G.F. McNulty, and W. Taylor. Growth problems for avoidable words, Theoret. Comput. Sci. 69 (1989), 319-345.

[2] D.R. Bean, A. Ehrenfeucht, and G.F. McNulty. Avoidable patterns in strings of symbols. Pacific J. of Math. 85 (1979), 261-294.

[3] J. Bell, T. L. Goh. Exponential lower bounds for the number of words of uniform length avoiding a pattern. Inform. and Comput. 205 (2007), 1295-1306.

[4] F. Blanchet-Sadri, B. Woodhouse. Strict bounds for pattern avoidance. Theor. Comput. Sci. 506 (2013), 17-27. 
[5] J. Cassaigne. Motifs évitables et régularité dans les mots. Thèse de Doctorat, Université Paris VI, Juillet 1994.

[6] D. Gonçalves, M. Montassier, and A. Pinlou. Entropy compression method applied to graph colorings. arXiv: 1406.4380

[7] R. Kolpakov and M. Rao: On the number of Dejan words over alphabets of 5, 6, 7, 8, 9 and 10 letters. Theor. Comput. Sci. 412(46) (2011), 6507-6516.

[8] M. Lothaire. Algebraic Combinatorics on Words. Cambridge Univ. Press (2002).

[9] J. Miller. Two notes on subshifts. Proc. Amer. Math. Soc. 140 (2012), 1617-1622.

[10] P. Ochem. A generator of morphisms for infinite words. RAIRO: Theoret. Informatics Appl. 40 (2006), 427-441.

[11] P. Ochem and A. Pinlou. Application of entropy compression in pattern avoidance. Electron. J. Combinatorics. 21(2) (2014), \#RP2.7.

[12] D. I. Piotkovskii. On the growth of graded algebras with a small number of defining relations. Uspekhi Mat. Nauk. 48:3(291) (1993), 199-200.

[13] N. Rampersad. Further applications of a power series method for pattern avoidance. Electron. J. Combinatorics. 18(1) (2011), \#P134.

[14] A.I. Zimin. Blocking sets of terms. Math. USSR Sbornik 47(2) (1984), 353-364. English translation. 